
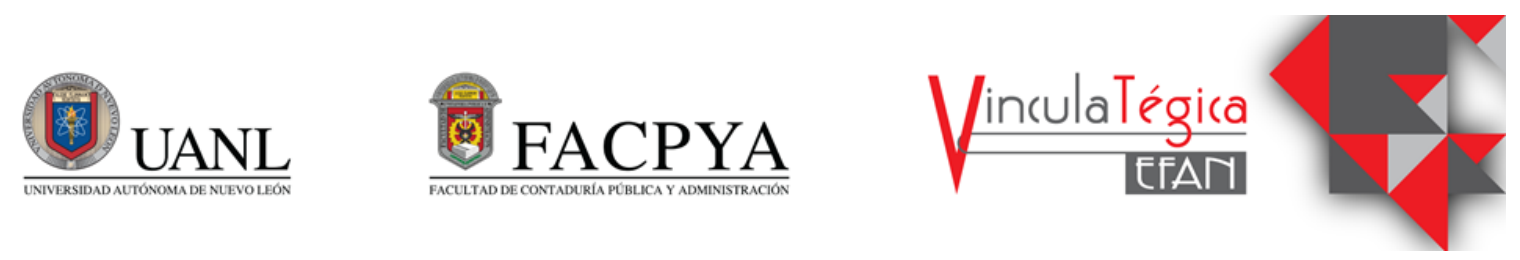

\title{
Factores que inciden en la satisfacción de los usuarios de sistemas de información en las organizaciones
}

\author{
Betzacarías Báez Vázquez y Manuel Alexis Vázquez Zacarías² \\ ${ }^{1}$ Universidad Autónoma de Nuevo León, betzacarias.baezv@uanl.edu.mx, Pedro de Alba S/N, Niños Héroes, \\ Ciudad Universitaria, San Nicolás de los Garza, N.L, 8341046301 \\ ${ }^{2}$ Universidad Autónoma de Nuevo León, manuel.vazquezzcr@uanl.edu.mx, Pedro de Alba S/N, Niños \\ Héroes, Ciudad Universitaria, San Nicolás de los Garza, N.L, 8182591215
}

Información del artículo revisado por pares

Fecha de aceptación: junio-2021

Fecha de publicación en línea: diciembre-2021

DOI: https://doi.org/10.29105/vtga7.2-45

\section{Resumen}

La presente investigación estudia el vínculo existente entre los factores de calidad del sistema, calidad de la información, calidad del servicio, utilidad y facilidad de uso en la satisfacción de los usuarios de sistemas de información en las organizaciones. Se busca desarrollar un modelo teórico sustentado en la literatura actualizada sobre el tema para su aplicación en futuras investigaciones.

Palabras clave: Factores de éxito, Satisfacción del usuario, Sistemas de Información.

\section{INTRODUCCIÓN}

Los Sistemas de Información (SI) son uno de los principales componentes para las organizaciones debido a que ofrecen la capacidad de reunir, procesar, distribuir y compartir datos e información de forma integrada y oportuna (Abrego-Almazán, Sánchez-Tovar, \& Medina-Quintero, 2017). De igual manera, permiten a los trabajadores mejorar los procesos de gestión y del manejo de la información dando como resultado un impacto positivo en la productividad y
Abstract

This research studies the link between the factors of system quality, information quality, service quality, usefulness and ease of use in the satisfaction of users of information systems in organizations. It seeks to develop a theoretical model based on the updated literature on the subject for its application in future research.

Keywords: Success factors, User satisfaction, Information Systems.

JEL: O33, O39, M15.

competitividad de las empresas (Ynzunza \& Izar, 2011).

Las ventajas que otorgan los SI conllevan a que con el paso del tiempo las organizaciones cada vez dependan más de estas herramientas para llevar a cabo sus actividades diarias (Gómez \& Suárez, 2012). Debido a que el uso e implementación de un SI en una organización requiere de una inversión en recursos y tiempo es importante que este cubre las necesidades de los usuarios o trabajadores, de ahí surge la importancia de evaluar si 
cumple con el propósito para el cual fue creado, es decir, si el SI es exitoso (DeLone \& McLean, 2016).

Debido a que con el transcurso del tiempo los SI han evolucionado, cambiando y adaptándose a las nuevas necesidades de los usuarios la comprensión de éxito de SI de igual manera se ha modificado. Por ello, los investigadores en el tema han planteado distintas formas y enfoques para describir la historia de los SI (Petter, DeLone \& McLean, 2012).

Para definir cómo han ido evolucionando los SI algunos investigadores se han enfocado en las características de las tecnologías utilizadas para el uso de los SI tales como el hardware, el internet o el uso de dispositivos portátiles (Dahlbom, 1996; Jessup \& Valacich, 1999). Sin embargo, estos cambios se centran en la parte evolutiva de la tecnología que se utiliza para el uso de SI, por tanto, una mejor manera de hacerlo es establecer eras conforme al tipo de SI que han predominado por un determinado periodo de tiempo (DeLone \& McLean, 2016).

Mediante la revisión de la literatura se han logrado establecer 5 eras de los SI; la era del procesamiento de datos (1950-1960), la del informe de gestión y apoyo a la toma de decisiones (1960- 1980), la era de la computación estratégica y personal (19801990), la del sistema empresarial y conexión en red (1990-2000) y la era focalizada en el cliente (2000 y más) (DeLone \& McLean, 2016).

En primer término, la era de procesamiento de datos estuvo presente en las décadas de los años 50's y 60's. En este periodo de tiempo, las computadoras eran prácticamente calculadoras muy sofisticadas utilizadas únicamente por ingenieros altamente capacitados para su uso, por ello el número de usuarios era muy reducido (Hirschheim \& Klein, 2011).

Por otra parte, durante las décadas de los años 60's y 70’s se ubica la era del informe de gestión y apoyo a la toma de decisiones. En este periodo de tiempo los trabajadores en las organizaciones comenzaron a utilizar las computadoras para automatizar labores administrativas mejorando su rendimiento, durante esta era el uso de SI comenzó a ser de utilidad para los gerentes en los procesos de toma de decisiones (Dahlbom, 1996).

Posteriormente, se encuentra la era de la computación estratégica y personal ubicada entre la década de los años 80's y 90's. La principal característica en este periodo es que las organizaciones comenzaron a utilizar los SI para alcanzar sus objetivos o metas debido a que los SI comenzaron a simplificarse y a que la percepción de los beneficios que brindaban a las empresas comenzó a incrementarse (Dahlbom, 1996). En este punto se vislumbró la necesidad de alinear los SI con los objetivos de las organizaciones y la necesidad de medir el éxito de los SI (DeLone \& McLean, 2016).

Por otro lado, la era del sistema empresarial y la conexión en red se ubicó entre los años 1990’s y 2000. Durante este periodo cambió la manera en la que las organizaciones utilizaban los SI debido a que comenzó la comunicación cliente-servidor y el uso de redes de Internet permitiendo compartir datos entre los usuarios o distintos departamentos de la organización (Dahlbom, 1996).

Finalmente, la era focalizada en el cliente cuyo inicio fue a principios del año 2000 y continúa hasta el día de hoy. En esta era los clientes interactúan más con SI que con los trabajadores de una organización, pueden realizar pedidos, dar seguimiento a una solicitud o recibir información sin la necesidad de interactuar físicamente con una persona (DeLone \& McLean, 2016). En la figura 1 se muestran de manera gráfica las eras de SI con el paso del tiempo y sus principales características. 
Figura 1 Eras de los SI a través de los años

\begin{tabular}{|c|c|c|c|c|}
\hline $\begin{array}{c}\text { Era de } \\
\text { procesamiento de } \\
\text { datos } \\
\end{array}$ & $\begin{array}{l}\text { Era de informe de } \\
\text { gestión y apoyo a la } \\
\text { toma de decisiones }\end{array}$ & $\begin{array}{l}\text { Era de la computación } \\
\text { estratégica y personal }\end{array}$ & $\begin{array}{c}\text { Era del sistema } \\
\text { empresarial y } \\
\text { conexión en red }\end{array}$ & $\begin{array}{l}\text { Era focalizada } \\
\text { en el cliente }\end{array}$ \\
\hline $1050-1960$ & 1960-1980 & $1980-1990$ & $1990-2000$ & $2000 \mathrm{ym}$ más \\
\hline $\begin{array}{l}\text { "Las } \\
\text { computadoras eran } \\
\text { básicamente unas } \\
\text { calculadoras muy } \\
\text { sofisticadas. } \\
\text { "Las } \\
\text { computadoras } \\
\text { únicamente eran } \\
\text { utilizadas por } \\
\text { ingenieros } \\
\text { capacitados. } \\
\text { "El número de } \\
\text { personas que } \\
\text { utilizaban las } \\
\text { computadoras era } \\
\text { sumamente } \\
\text { reducido. }\end{array}$ & $\begin{array}{l}\text { "Los trabajadores } \\
\text { en las empresas } \\
\text { comenzaron a } \\
\text { utilizar más las } \\
\text { computadoras. } \\
\text { *Más individuos } \\
\text { dentro de las } \\
\text { empresas se } \\
\text { expusieron al uso de } \\
\text { las computadoras. } \\
\text { "Los gerentes } \\
\text { comenzaron a } \\
\text { utilizar la } \\
\text { información } \\
\text { resultante de estos } \\
\text { sistemas en su toma } \\
\text { de decisiones. }\end{array}$ & $\begin{array}{l}\text { "Los gerentes y las } \\
\text { organizaciones en } \\
\text { general } \\
\text { comenzaron a } \\
\text { utilizar los SI para } \\
\text { lograr alcanzar sus } \\
\text { metas. } \\
\text { "Los usuarios cada } \\
\text { e vez necesitaban } \\
\text { menos } \\
\text { entrenamiento para } \\
\text { el uso de SI. } \\
\text { "Los usuarios } \\
\text { podian crear } \\
\text { beneficios para sus } \\
\text { empresas a través } \\
\text { de su uso de SI. }\end{array}$ & $\begin{array}{l}\text { *Se inició la } \\
\text { comunicación } \\
\text { cliente-servidor. } \\
\text { *Se comenzaron a } \\
\text { utilizar las redes de } \\
\text { internet. } \\
\text { *Cambió la forma } \\
\text { en que las } \\
\text { organizaciones } \\
\text { utilizaban sus SI. }\end{array}$ & 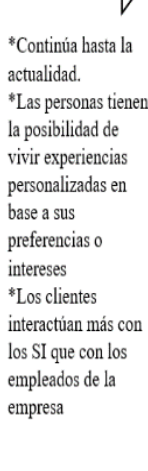 \\
\hline $\begin{array}{l}\text { Fuente: El } \\
\text { (2016). }\end{array}$ & oración p & opia a part & & \\
\hline
\end{tabular}

El impacto que tienen los Sistemas de Información (SI) en las organizaciones ha sido objeto de estudio desde hace tiempo (Solano, García, \& Bernal, 2014). En la actualidad, la medición del éxito de SI se mantiene como una de las principales preocupaciones de investigadores, profesionales y administradores (Hasan et al., 2017). Si bien dentro de la literatura no existe un consenso sobre la definición de éxito en SI (Martins et al., 2019), la satisfacción del usuario ha sido ampliamente considerada como la principal medida de éxito (Al-Samarraie, Teng, Alzahrani, \& Alalwan, 2018).

Las investigaciones enfocadas en la satisfacción del usuario han considerado factores agrupados a nivel de calidad, personal y organizacional, así como múltiples relaciones entre sí (Kofahe, Hassan, \& Mohamad, 2019; Rouibah, Dihani, \& AlQirim, 2020). Tomando en cuenta los dos primeros grupos, dentro de los factores de calidad se encuentran la calidad del sistema, calidad de la información y calidad del servicio, mientras que dentro de los factores de nivel personal se encuentran la utilidad y la facilidad de uso (Almaiah \& Alismaiel, 2019).

Investigaciones previas han encontrado que es necesaria la calidad en el sistema para tener satisfacción en el usuario (Abrego-Almazán et al., 2017; Song, Migliaccio, Wang, \& Lu, 2017; Juárez, Quintero, \& Almazán, 2018). Por otro lado, la calidad de la información es indispensable en la satisfacción del usuario (Kilsdonk, Peute, \& Jaspers, 2017; Utama, Purnomosidhi, \& Andayani, 2017; Kofahe et al., 2019). Inclusive, dentro de la literatura se encuentran investigaciones que indican que la calidad en el servicio influye en la satisfacción del usuario (Jeon \& Jeong, 2017) (Song et al., 2017; Haddad, 2018).

En cuanto a los factores de carácter personal, se ha determinado que la utilidad percibida incide en la satisfacción del usuario (Tandon, Kiran, \& Sah, 2016; Shah \& Attiq, 2016; Wang \& Song., 2017; Baki, Birgoren, \& Aktepe, 2018; Xu \& Du, 2018; Rouibah et al., 2020). De igual manera, dentro de la literatura existen estudios que han comprobado la relación entre la facilidad de uso y la satisfacción del usuario (Tandon, et al., 2016; Shah \& Attiq, 2016).

Por tanto, existe un conflicto de corrientes debido a la falta un acuerdo que nos permita comprender el rol y la importancia de los factores calidad del sistema, calidad de la información, calidad del servicio, utilidad y facilidad de uso en la satisfacción del usuario. $\mathrm{Y}$ derivado de ello surge la siguiente pregunta de investigación:

¿Qué factores clave tienen incidencia en la satisfacción de los usuarios de SI?

Para dar respuesta a la pregunta de investigación planteada, el objetivo de este estudio es desarrollar un modelo teórico que vincule los factores calidad del sistema, calidad de la información, calidad del servicio, utilidad y facilidad de uso con la satisfacción del usuario.

\section{MARCO TEÓRICO}

Primeramente y para dar fundamento teórico a lo que se pretende investigar, se parte de las referencias existentes de trabajos de investigación realizados con anterioridad cuyo objetivo ha sido el estudio de la satisfacción 
del usuario de SI en distintos contextos.

La idea de que la satisfacción del usuario es el principal indicador de éxito en un sistema de información es ampliamente aceptada dentro del campo de estudio (DeLone \& McLean, 2016). Ejemplo de lo anterior son los estudios que se han realizado en años recientes. A finales de la década pasada, se llevó a cabo una investigación buscando determinar qué factores de carácter organizacional y de motivaciones humanas influyen en la satisfacción de los usuarios, considerando a esta última variable como principal medida de éxito de SI en organizaciones públicas (Rouibah, Hamdy, \& Al-Enezi, 2009).

Posteriormente, en los inicios de la presente década, se elaboró un trabajo de investigación dirigido a validar el modelo de éxito de D\&M debido a que generalmente los trabajos que utilizan como base dicho modelo realizan un análisis a nivel individual, en esta ocasión se aplicó a un sitio web y en un nivel de análisis organizacional (Pérez, 2010). En dicho estudio $\mathrm{y}$ al igual que en el modelo original la satisfacción del usuario se mantiene como la principal medida de éxito de un SI.

Más adelante, se realizó un estudio en el cual se propuso un modelo integrado para evaluar el éxito de SI a partir del modelo D\&M y el Technology acceptance model (TAM) en dónde definió a la satisfacción del usuario como la respuesta positiva o negativa resultante de las expectativas obtenidas a partir del uso del consumo (Zaied, 2012). Si bien, en el anterior estudio se tomó a la actitud de uso como medida final de éxito de SI en su modelo propuesto, la satisfacción del usuario figura como precursor de esta, por tanto, se mantiene como una de las principales medidas de éxito.

En años más recientes los estudios sobre el éxito de SI han continuado vigentes y adaptándose a las nuevas tendencias tecnológicas continuando la satisfacción del usuario como una de las principales claves para el éxito de SI. Referente a lo mencionado, se llevó a cabo una investigación en dónde se propuso una mejora al modelo D\&M para su aplicación en el comercio electrónico, se encuestaron a 288 consumidores recurrentes y se encontró que la satisfacción del usuario juega un papel fundamental en el éxito de sistemas de este tipo (Rouibah, Lowry \& Almutairi, 2015).

Las investigaciones que abordan el constructo satisfacción del usuario en SI se han aplicado en distintos tipos de organizaciones y en diferentes industrias. En 2016 se elaboró un estudio para explorar las percepciones de los médicos y directores de hospitales con respecto a los factores clave de éxito de un sistema de gestión del conocimiento clínico para ello se realizó una encuesta en cuatro centros médicos de Taiwán y se aplicaron un total de 340 cuestionarios, incluidos 15 para directores de hospitales y 70 para médicos de cada hospital, los resultados muestran que la satisfacción tiene efectos positivos sobre la percepción del desempeño del SI, es decir, incide en su éxito. (Chang, Hsieh, \& Chen, 2016).

De igual manera, la industria hotelera no queda exenta del uso de SI para la gestión de sus actividades, en este sentido, la satisfacción del usuario tiene un papel fundamental en el éxito de SI para este tipo de organizaciones. Tal y como se demostró en una investigación en donde se estudiaron los determinantes de éxito en un sitio web de alojamientos (Jeon \& Jeong, 2017).

Posteriormente, se realizó un estudio buscando determinar qué factores influyen en el éxito de sistemas de gestión de aprendizaje y se tomó a la satisfacción del usuario como principal medida de éxito ya que se ha destacado dentro de la literatura debido a que pronostica en gran medida el éxito de sistemas en donde una mayor tasa de satisfacción de los usuarios aumenta los beneficios para los estudiantes (Haddad, 2018).

En SI de gestión para la educación, la satisfacción del usuario se mantiene como un constructo fundamental para el éxito del SI. Ejemplo de lo anterior es una investigación 
elaborada recientemente en dónde se busca comprender qué factores impactan en la satisfacción de los estudiantes con respecto al uso de sistemas gestores de educación, los hallazgos del estudio vislumbran que tal y como afirman los modelos de éxito de SI la satisfacción del usuario tiene un papel fundamental (Martins et al., 2019).

Dentro de la literatura también se puede encontrar que la satisfacción del usuario continúa siendo un tema vigente. En la actualidad y a raíz de la necesidad del uso de plataformas de educación en línea en China por la pandemia de COVID-19 se considera a la de satisfacción del usuario como principal objeto de estudio (Chen et al., 2020).

A continuación, se busca explicar y fundamentar teóricamente la relación existente entre las variables de estudio. Comenzando con la calidad del sistema, se define como como el grado en que los usuarios perciben un SI cumple con las expectativas que se tienen de él (Abrego-Almazán et al., 2017). La calidad del sistema también se puede referir a la capacidad que tiene un SI para brindar a sus usuarios confiabilidad, usabilidad y tiempos de respuesta adecuados para las actividades que esté destinado a desempeñar (Song et al., 2017). De igual manera, se refiere a la percepción global del usuario de SI en dónde se consideran cuestiones como la capacidad del sistema para ser utilizado de manera sencilla además de proporcionar al usuario todas las funciones que este requiera (Martins et al., 2019).

En cuanto a la relación de la calidad del sistema con la satisfacción del usuario, el estudio realizado por Chang et al., (2016) en donde se exploraron las percepciones de médicos y directores de hospitales con respecto a los factores de éxito claves en un SI encargado de la gestión de conocimiento clínico. Se aplicaron un total de 340 cuestionarios en hospitales de Taiwán y a través del análisis de mínimos cuadrados parciales (PLS) y una técnica de modelado de ecuaciones estructurales (SEM) basada en componentes la cual resulta muy adecuada para evaluar modelos predictivos complejos como el utilizado por los investigadores. Se comprobó que tanto para los directivos $(\beta$ $=.521 ; \mathrm{p}<.001)$ como para los médicos $(\beta$ $=.456 ; \mathrm{p}<.001)$ la calidad del sistema tiene una influencia positiva sobre la satisfacción del usuario.

De igual manera, en la investigación realizada por Ábrego-Almazán et al., (2017) se encontró a través de la revisión de la literatura que la calidad del sistema se relaciona positivamente con la satisfacción del usuario. Los autores utilizaron ecuaciones estructurales en su modelo mediante la técnica estadística de mínimos cuadrados parciales (partial least squares, [PLS]) lo cual arrojó el siguiente resultado: $(\beta=.291 ; \mathrm{p}<.001)$. El estudio se realizó mediante la aplicación de 133 cuestionarios a empresas del estado de Tamaulipas, México.

Posteriormente Laumer, Maier y Weitzel (2017) realizaron un estudio mixto basado en el modelo de éxito de DeLone \& McLean en el cual realizaron 34 entrevistas a empleados y encuestaron a un total de 247 usuarios de un SI encargado de administrar contenido empresarial, en su investigación encontraron que la calidad del sistema influye positiva y significativa en la satisfacción de los usuarios $(\beta=.511 ; \mathrm{p}<.001)$.

Aunado a lo anterior, otro estudio realizado por Muda y Erlina (2019) tuvo como propósito examinar el efecto de la calidad del sistema y de la información en la satisfacción de los usuarios de un SI para gestión contable en indonesia encontró que existe una relación positiva y significativa entre la calidad del sistema y la satisfacción del usuario $(\beta=.316$; $\mathrm{p}<.001)$, dichos resultados se obtuvieron a través de la aplicación de 209 cuestionarios a usuarios de dicho SI y los datos recolectados se analizaron utilizando el método PLS y una técnica de SEM.

La relación entre la calidad del sistema y la satisfacción del usuario se encuentra planteada con el suficiente sustento teórico dentro de la literatura (Chang et al., 2016; Song et al., 
2017; Kilsdonk, et al., 2017; Utama et al., 2017; Andarwati, Nirwanto, \& Darsono, 2018; Haddad, 2018; Juárez et al., 2018; Kofahe et al., 2019).

Por otra parte, y tomando en cuenta el segundo factor de calidad considerado para la presente investigación. La calidad de la información se refiere a la calidad de las salidas de un SI y su adecuada presentación para su uso en un determinado contexto de aplicación (Pôças et al., 2014). La calidad de la información también se puede definir como " Una medida de la calidad de los productos (SI): a saber, la calidad de la información que el sistema produce en informes y en pantalla" (Gable, Sedera \& Chan, 2008, p. 389). De igual manera, se refiere a la percepción del usuario sobre las salidas del SI en cuestiones como; relevancia, consistencia, exactitud y oportunidad (DeLone \& McLean, 2003).

La relación entre la calidad de la información y la satisfacción del usuario se encuentra plasmada y sustentada dentro de la literatura en una investigación realizada por Utama et al., (2017) quienes estudiaron la influencia de distintos factores sobre la satisfacción de los usuarios de un SI financiero regional en indonesia. Recopilando información de un total de 147 miembros del personal de gestión financiera que utiliza dicho SI y mediante el uso de SEM lograron determinar que la calidad de la información influye positiva y significativamente en la satisfacción del usuario $(\beta=.617 \mathrm{p}<.001)$.

Por otra parte, Song et al., (2017) en su estudio plasmaron y comprobaron una hipótesis similar relacionando la calidad de la información con la satisfacción del usuario. En su trabajo estudiaron la satisfacción de los usuarios de un SI para la construcción de las industrias AEC en China, para obtener los resultados se distribuyeron 280 cuestionarios de los cuales 113 fueron respondidos validados y tomados en cuenta. Mediante el método PLS se logró comprobar que la calidad de la información incide en la satisfacción del usuario $(\beta=.410 \mathrm{p}<.01)$.
Por otro lado, Santa, MacDonald y Ferrer (2019) realizaron una investigación para estudiar los factores que influyen en la satisfacción de los usuarios de SI para la gestión gubernamental en Arabia Saudita. Con base en una muestra de 209 cuestionarios aplicados a usuarios de aplicaciones de gobierno electrónico en empresas locales en Arabia Saudita y utilizando el método SEM se determinó que la calidad de la información tiene un efecto positivo sobre la satisfacción del usuario $(\beta=.369 p<.01)$.

En tiempos más recientes, un estudio realizado por Shim y Jo (2020) aplicó el modelo actualizado de DeLone y McLean para el éxito de los SI en el portal nacional de información de salud en Corea del Sur. Los autores realizaron una encuesta en línea en el año 2017 a 506 adultos usuarios de dicho portal y mediante un análisis factorial confirmatorio, una regresión jerárquica de mínimos cuadrados ordinarios y un enfoque de arranque para una prueba de mediación comprobaron que la calidad de la información tiene un impacto positivo y significativo en la satisfacción del usuario $(\beta=.49 \mathrm{p}<.001)$.

Aunado a lo anterior, otras investigaciones previas establecen y sustentan una relación existente entre la calidad de la información y la satisfacción del usuario (Kilsdonk et al., 2017; Haddad, 2018; Kofahe et al., 2019).

Por otro lado, la calidad del servicio se define como la capacidad del departamento encargado de dar soporte al SI para brindar ayuda a los usuarios (DeLone \& McLean, 2016). Parasuraman, Zeithaml, \& Berry (1985) mencionan que se refiere a la distancia que existe entre las expectativas del servicio que se espera obtener y el servicio que realmente se percibe.

La relación entre la calidad del servicio y la satisfacción del usuario fue abordada en una investigación citada con anterioridad realizada por Ábrego-Almazán et al., (2017) se comprobó que la calidad del servicio tiene una relación positiva y significativa con la satisfacción del usuario $(\beta=.310 \mathrm{p}<.001)$. Lo 
anterior se obtuvo mediante la recopilación de información a través de 133 cuestionarios aplicados a empresas pertenecientes al estado de Tamaulipas en México y utilizando el método estadístico PLS.

En otro estudio empírico realizado por Ameen, Al-ali, Isaac y Mohammed (2020) de igual manera se determinó que existe una relación positiva y significativa entre la calidad del servicio y la satisfacción del usuario $(\beta=.573 \mathrm{p}<.001)$. Dicho resultado lo obtuvieron tras proponer un modelo teórico basado en el modelo de éxito de DeLone y McLean para estudiar SI gubernamentales en los Estados Unidos de América (EUA), recopilaron información de 147 empleados de organizaciones públicas a través de un cuestionario y para la obtención de los resultados utilizaron el modelado SEM mediante regresión PLS.

La relación planteada entre la calidad del servicio y la satisfacción del usuario continúa siendo un tema de actualidad, a raíz de la pandemia por COVID-19 en el año 2020 la enseñanza tradicional presencial ha cambiado y se ha mudado a plataformas en línea. Respecto a lo anterior, se llevó a cabo un estudio con la intención de estudiar la satisfacción de los usuarios de plataformas online para la educación en china (Chen et al., 2020). En dicho trabajo se recopiló información a través de un cuestionario aplicado a usuarios de las plataformas para aprendizaje más representativas de la región (Ding Ding, Tencent Meeting, Tencent Class, Chaoxing Learning y MOOC) y dentro de sus resultados lograron determinar que la calidad del servicio tiene una influencia positiva y significativa en la satisfacción del usuario $(\beta$ $=.389 \mathrm{p}<.001$ ).

Por otra parte, Alrwashdeh et al., (2020) llevaron a cabo una investigación para estudiar la satisfacción de los usuarios en el contexto de los operadores móviles en el norte de Chipre. Se recopiló información a través de 500 cuestionarios aplicados en la Girne American University y los datos recolectados se analizaron mediante el modelado SEM utilizando el Paquete Estadístico para las Ciencias Sociales (SPSS) y el Análisis de Estructuras de Momentos (AMOS) obteniendo entre sus resultados que la calidad del servicio influye en la satisfacción del usuario $(\beta=.829 \mathrm{p}<.001)$.

$\mathrm{Al}$ igual que en los estudios anteriormente mencionado, la relación entre calidad del servicio y satisfacción del usuario se encuentra planteada y sustentada teóricamente en otras investigaciones (Jeon \& Jeong, 2017) (Kilsdonk et al., 2017; Utama et al., 2017; Haddad, 2018; Kofahe et al., 2019).

En cuanto a los constructos tomados de los factores de nivel personal, primeramente, se aborda la utilidad la cual se define como la medida en la cual un individuo considera que el uso de un sistema en específico incrementará su desempeño en el trabajo (Davis, 1985). Este constructo también ha sido definido como el interés por parte del usuario por utilizar una tecnología y que, a su vez, el uso de ella mejorará el desempeño laboral (Ozturk, 2016).

En cuanto a la facilidad de uso, se define como el grado en que una persona cree que usar una tecnología en particular no requiere esfuerzo (Davis, 1989). De igual manera, en este mismo contexto se refiere a el grado en el que un SI se percibe como fácil de entender y operar (Deb \& Agrawal, 2017). En otra investigación la facilidad de uso se definió como la medida en que un sistema en específico es fácil de usar o no (Dong, Chang, Wang, \& Yan, 2017).

Con respecto a las relaciones planteadas entre la utilidad y la facilidad de uso con la satisfacción del usuario, estas fueron abordadas por Shah y Attiq (2016) quienes estudiaron el impacto de la calidad de la tecnología sobre la satisfacción del usuario en el contexto del aprendizaje en línea. Los autores aplicaron un cuestionario en línea a un total de 1338 estudiantes del sistema de educación virtual en Pakistán y a través de un análisis de correlación determinaron que tanto la utilidad $(\beta=.18 \mathrm{p}<.05)$ como la facilidad de 
uso $(\beta=.75 \mathrm{p}<.05)$ influyen positivamente en la satisfacción de los usuarios.

En la investigación de Tandon et al., (2016) se estudiaron los factores clave que influyen en la satisfacción de los usuarios de ecommerce en la India. Mediante el método de correlación y el análisis de datos recolectados de 300 cuestionarios aplicados de manera personal y 65 mediante correo electrónico desde junio de 2014 hasta febrero 2014 a consumidores del norte de India que tuvieron alguna experiencia de compra en línea se logró determinar que existe una relación positiva entre la facilidad de uso y la satisfacción del usuario $(\beta=.149 \mathrm{p}<.01)$.

Por otro lado, en el trabajo realizado por Wang y Song (2017) se determinó que la utilidad incide en la satisfacción del usuario $(\beta$ $=.184 \mathrm{p}<.05)$. Para llegar a esa conclusión los autores estudiaron los factores que inciden en el éxito de SI utilizados en la industria de la construcción en China, recopilaron información a través de cuestionarios aplicados a 118 ingenieros usuarios de dicho SI y utilizaron PLS para realizar las inferencias sobre sus relaciones planteadas.

Como un sustento más de la relación entre la utilidad y la satisfacción se puede mencionar el estudio realizado por $\mathrm{Xu}$ y $\mathrm{Du}$ (2018) en donde se buscó identificar qué factores influyen en la satisfacción y la lealtad de los usuarios de bibliotecas digitales de universidades chinas, a través de la aplicación de cuestionarios a 426 sujetos de estudio y al análisis de datos recolectados mediante el modelado SEM lograron determinar que la utilidad influye positiva y significativamente en la satisfacción de los usuarios $(\beta=.84$ $\mathrm{p}<.05)$.

En años más recientes se llevó a cabo una investigación por Normelindasari y Solichin (2020) quienes estudiaron los factores que inciden en la satisfacción de los usuarios de una aplicación web para brindar servicios académicos a los estudiantes de la Universidad Budi Luhur en Yakarta, Indonesia. Para realizar su estudio recopilaron información mediante un cuestionario aplicado a 380 estudiantes de dicha universidad, posteriormente obteniendo dentro de sus resultados que la utilidad tiene un efecto positivo y significativo sobre la satisfacción del usuario $(\beta=.628 \mathrm{p}<.001)$.

En la tabla 1 se presenta a manera de resumen las variables estudiadas y los estudios que sustentan, la relación con la satisfacción del usuario y su principal su aporte.

\begin{tabular}{|c|c|c|}
\hline \multicolumn{3}{|c|}{ Tabla 1} \\
\hline Relación & Autores & Hallazgos \\
\hline $\begin{array}{lr}\text { Calidad del } \\
\text { sistema } \\
\text { satisfacción }\end{array}$ & $\begin{array}{l}\text { (Chang et al,, 2016; } \\
\text { Song et al., 2017; } \\
\text { Kilsdonk, et al,. 2017; } \\
\text { Utama et al,, 2017; } \\
\text { Andarwati, Nirwanto, \& } \\
\text { Darsono, 2018; Haddad, } \\
\text { 2018; Juáreze tal., 2018; } \\
\text { Kofahe et al., 2019). }\end{array}$ & $\begin{array}{l}\text { Relación } \\
\text { positiva }\end{array}$ \\
\hline $\begin{array}{l}\text { Calidad de la } \\
\text { información - } \\
\text { satisfacción }\end{array}$ & $\begin{array}{l}\text { (Kilsdonk et al,, 2017; } \\
\text { Haddad, 2018; Kofahe et } \\
\text { al., 2019). }\end{array}$ & $\begin{array}{l}\text { Relación } \\
\text { positiva }\end{array}$ \\
\hline $\begin{array}{l}\text { Calidad del } \\
\text { servicio } \\
\text { satisfacción }\end{array}$ & $\begin{array}{l}\text { (Jeon \& Jeong, 2017) } \\
\text { (Kilsdonk et al., 2017; } \\
\text { Utama et al., 2017; } \\
\text { Haddad, 2018; Kofahe et } \\
\text { al., 2019). }\end{array}$ & $\begin{array}{l}\text { Relación } \\
\text { positiva }\end{array}$ \\
\hline $\begin{array}{l}\text { Utilidad } \\
\text { satisfacción }\end{array}$ & $\begin{array}{l}\text { Shah \& Attiq, 2016; } \\
\text { Wang \& Song, 2017; Xu } \\
\text { \& Du, 2018; } \\
\text { Normelindasari } \\
\text { Solichin, 2020) }\end{array}$ & $\begin{array}{l}\text { Relación } \\
\text { positiva }\end{array}$ \\
\hline $\begin{array}{ll}\text { Facilidad de } \\
\text { uso }\end{array}$ & $\begin{array}{l}\text { (Shah \& Attiq, 2016; } \\
\text { Tandon et al., 2016) }\end{array}$ & $\begin{array}{l}\text { Relación } \\
\text { positiva }\end{array}$ \\
\hline
\end{tabular}

Derivado de las investigaciones encontradas dentro de la literatura sobre el tema se concluye con el modelo teórico mostrado en la figura 2, en él se observan de manera gráfica tanto las variables independientes del estudio (calidad del sistema, calidad de la información, calidad del servicio, utilidad y facilidad de uso) como su relación con la variable dependiente (satisfacción). 
Figura 2 Modelo gráfico de la investigación

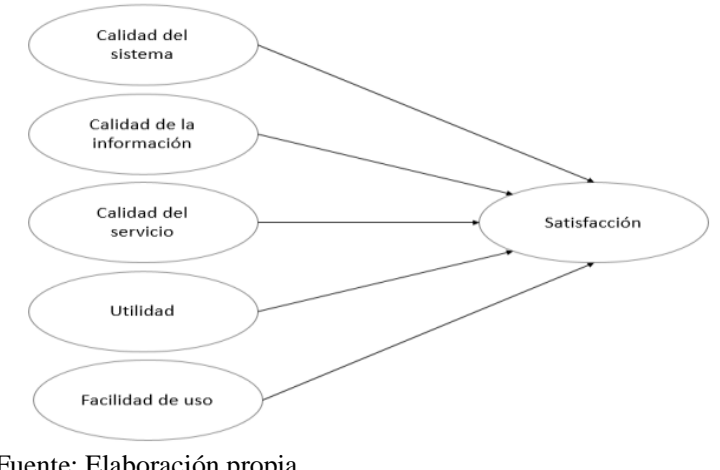

Fuente: Elaboración propia.

\section{CONCLUSIONES}

En el presente estudio se investigaron factores que inciden en la satisfacción de los usuarios de un sistema de información. Se hizo con el propósito de desarrollar un modelo teórico y así contribuir al desarrollo de estudios de este tipo.

De igual manera, en la presente investigación se planteó una pregunta de investigación la cual se logró dar respuesta estableciendo que tanto la calidad del sistema, la calidad de la información, la calidad del servicio, la utilidad y la facilidad de uso tienen un efecto positivo sobre la satisfacción del usuario de SI.

Además, se planteó un objetivo general el cual se logró realizar de manera satisfactoria. Lo anterior debido a que se mostró el vínculo entre las variables de estudio y se concluyó con un modelo teórico para explorar en futuras investigaciones.

Finalmente se debe mencionar las futuras líneas de investigación. Debido a que los beneficios del uso de SI no son exclusivos del sector privado, la modernidad y la renovación se puede dar en las Instituciones de Educación Superior (IES) a través del uso de SI (Garzón , 2015). En la actualidad, uno de los retos más desafiantes para las IES es el perfeccionamiento de la gestión universitaria, así como cada uno de los procesos que la integran (Acosta, Becerra, \& Jaramillo, 2017), lo cual se puede lograr mediante el uso de SI. Por lo tanto, resulta pertinente desarrollar estudios que busquen explicar la satisfacción del usuario en el contexto de IES pudiendo tomar como referencia el modelo planteado en el presente artículo. 


\section{REFERENCIAS}

Abrego-Almazán, D., Sánchez-Tovar, Y., \& Medina-Quintero, J. (2017). Influence of information systems in organizational results. Contaduría y Administración, 62(2), 321-338.

Abreu, J. (2012). La pregunta de investigación: alma del método científico. Monterrey, México: UANL.

Acosta, L., Becerra, F., \& Jaramillo, D. (2017). Sistema de Información Estratégica para la Gestión Universitaria en la Universidad de Otavalo (Ecuador). Formación universitaria., 10(2), 103112.

Almaiah, M., \& Alismaiel, O. (2019). Examination of factors influencing the use of mobile learning system: An empirical study. Education and Information Technologies, 24(1), 885-909.

Al-Mamary, Y., Shamsuddin, A., Hamid, A., \& Aziati, N. (2014). Factors affecting successful adoption of management information systems in organizations towards enhancing organizational performance. American Journal of Systems and Software, 2(5), 121-126.

Alrwashdeh, M., Jahmani, A., Ibrahim, B., \& Aljuhmani, H. (2020). Data to model the effects of perceived telecommunication service quality and value on the degree of user satisfaction and e-WOM among telecommunications users in North Cyprus. Data in brief. doi:https://doi.org/10.1016/j.dib.2019.104981

Al-Samarraie, H., Teng, B., Alzahrani, A., \& Alalwan, N. (2018). Learning continuance satisfaction in higher education: a unified perspective from instructors and students. Studies in Higher Education, 43(11), 2003-2019.

Ameen, A., Al-ali, D., Isaac, O., \& Mohammed, F. (2020). Examining relationship between service quality, user satisfaction, and performance impact in the context of smart government in UAE. International Journal of Electrical and Computer Engineering (IJECE), 10(6), 60266033.

Andarwati, M., Nirwanto, N., \& Darsono, J. (2018). Analysis of factors affecting the successof accounting information systems based on information technology on SME managementsas accounting informationend user. EJEFAS Journal, 97-102.

Baki, R., Birgoren, B., \& Aktepe, A. (2018). A Meta Analysis of Factors Affecting Perceived Usefulness and Perceived Ease of Use in the Adoption of E-Learning Systems. Turkish Online Journal of Distance Education, 19(4), 4-42.

Chang, S., Hsieh, P., \& Chen, H. (2016). Key success factors for clinical knowledge management systems: Comparing physician and hospital manager viewpoints. Technology and Health Care, 24(1), 297-306.

Chen, T., Peng, L., Yin, X., Rong, J., Yang, J., \& Cong, G. (2020). Analysis of user satisfaction with online education platforms in China during the COVID-19 pandemic. Healthcare, 8(3), 200226.

Davis, F. (1985). A technology acceptance model for empirically testing new end-user information systems: Theory and results. Tesis doctoral, Massachusetts Institute of Technology. 
Deb, M., \& Agrawal, A. (2017). Factors impacting the adoption of m-banking: understanding brand India's potential for financial inclusion. Journal of Asia Business Studies., 11(1), 22-40.

DeLone, W., \& McLean, E. (2016). Information systems success measurement. Foundations and Trends ${ }^{\circledR}$ in Information Systems, 2(1), 1-116.

Dong, X., Chang, Y., Wang, Y., \& Yan, J. (2017). Understanding usage of Internet of Things (IOT) systems in China: Cognitive experience and affect experience as moderator. nformation Technology \& People, 30(1), 117-138. doi:10.1108/ITP-11-2015-0272.

Gable, G., Sedera, D., \& Chan , T. (2008). Re-conceptualizing information system success: the ISimpact measurement model. Journal of the Association for Information Systems, 9(7), 1-32.

Garzón , C. (2015). Políticas públicas de inclusión de las tecnologías de la información y comunicación en la educación superior mexicana. Revista De Pedagogía, 92-107.

Gómez, A., \& Suárez, R. (2012). Sistemas de información herramientas prácticas para la gestión empresarial (4.aed.). Madrid, España: Alfaomega.

Haddad, F. (2018). Examining the effect of learning management system quality and perceived usefulness on student's satisfaction. Journal of Theoretical and Applied Information Technology, 96(23).

Hasan, M., Maarop, N., Samy, G., Baharum, H., Abidin, W., \& Hassan, N. (2017). Developing a success model of Research Information Management System for research affiliated institutions. In 2017 international conference on research and innovation in information systems (ICRIIS) (págs. 1-6). IEEE.

Hernández, R., Fernández, C., \& Baptista, P. (2010). Metodología de la investigación. (5 ed.). México, D.F.: McGraw Hill Interamericana Editores.

Ikram, M., Zulkarnain, Z., \& Alwie, A. (2019). El efecto de la facilidad de uso percibida, la utilidad, la satisfacción del cilente y la confienza en la intención de recompra (Estudio de caso sobre usuarios del sitio Lazada en Pekanbaru). Jurnal Tepak Manajemen Bisnis, 11(3), 599-618.

Jeon, M., \& Jeong, M. (2017). Customers' perceived website service quality and its effects on eloyalty. International Journal of Contemporary Hospitality Management, 29(1), 438-457.

Juárez, J., Quintero, J., \& Almazán, D. (2018). Calidad de los Sistemas de Información y su Impacto en la Satisfacción del Usuario. Red Internacional de Investigadores en Competitividad, 8(1), 644-659.

Kilsdonk, E., Peute, L., \& Jaspers, M. (2017). Factors influencing implementation success of guideline-based clinical decision support systems: A systematic review and gaps analysis. International journal of medical informatics, 56-64.

Kofahe, M., Hassan, H., \& Mohamad, R. (2019). Factors affecting successful implementation of governmento financial management information system (GFMIS) in Jordan public sector: A proposed framework. International Journal of Accounting, 4(20), 32-44.

Laumer, S., Maier, C., \& Weitzel, T. (2017). Information quality, user satisfaction, and the manifestation of workarounds: a qualitative and quantitative study of enterprise content management system users. European Journal of Information Systems, 26(4), 333-360. 
Martins, J., Branco, F., Gonçalves, R., Au-Yong-Oliveira, M., Oliveira, T., Naranjo-Zolotov, M., \& Cruz-Jesus, F. (2019). Assessing the success behind the use of education management information systems in higher education. Telematics and Informatics, 38, 182-193.

Muda, I., \& Erlina, A. (2019). Influence of human resources to the effect of system quality and information quality on the user satisfaction of accrual-based accounting system. . Contaduria y administración, 64(2), 10-21.

Normelindasari, D., \& Solichin, A. (2020). Effect of system quality, information quality, and perceived usefulness on user satisfaction of webstudent applications to improve service quality for Budi Luhur University Students. In 4th International Conference on Management, Economics and Business, (págs. 77-82). Atlantis Press.

Ozturk, A. (2016). Customer acceptance of cashless payment systems in the hospitality industry. International Journal of Contemporary Hospitality Management, 18(4), 801-817.

Parasuraman, A., Zeithaml, V., \& Berry, L. (1985). A Conceptual Model of Service Quality and Its Implications for Future Research. Journal of Marketing, 49(4), 41-50.

Pérez, B. (2010). Validity of DeLone and McLean's model of information systems success at the web site level of analysis. EUA: Louisiana State University. Tesis inédita.

Pôças, I., Gonçalves, J., Marcos, B., Alonso, J., Castro, P., \& Honrado, J. (2014). Evaluating the fitness for use of spatial data sets to promote quality in ecological assessment and monitoring. International Journal of Geographical Information Science, 28(11), 2356-2371.

Rizan, M., Haro, A., \& Rahmadhina, B. (2019). Pengaruh perceived ease of use dan service quality terhadap customer satisfaction dengan trust sebagai intervening (studi kasus pada pengguna grab di wilayah jakarta). Jurnal Riset Manajemen Sains Indonesia, 10(2), 337-355.

Rouibah, K., Dihani, A., \& Al-Qirim, N. (2020). Critical Success Factors Affecting Information System Satisfaction in Public Sector Organizations: A Perspective on the Mediating Role of Information Quality. Journal of Global Information Management, 28(3), 77-98.

Rouibah, K., Hamdy, H., \& Al-Enezi, M. (2009). Effect of management support, training, and user involvement on system usage and satisfaction in Kuwait. Industrial Management \& Data Systems.

Rouibah, K., Lowry, P., \& Almutairi, L. (2015). Dimensions of business-to-consumer (B2C) systems success in Kuwait: Testing a modified DeLone and McLean IS success model in an ecommerce context. Journal of Global Information Management (JGIM), 23(3), 41-71. doi:10.4018/JGIM.2015070103

Santa, R., MacDonald, J., \& Ferrer, M. (2019). The role of trust in e-Government effectiveness, operational effectiveness and user satisfaction: Lessons from Saudi Arabia in e-G2B. Government Information Quarterly, 36(1), 39-50.

Shah, H., \& Attiq, S. (2016). Impact of technology quality, perceived ease of use and perceived usefulness in the formation of consumer's satisfaction in the context of e-learning. Abasyn Journal of Social Sciences, 9(1), 124-140. 
Shah, H., \& Attiq, S. (2016). Impact of technology quality, perceived ease of use and perceived usefulness in the formation of consumer's satisfaction in the context of e-learning. Abasyn Journal of Social Sciences, 9(1), 124-140.

Shim, M., \& Jo, H. (2020). What quality factors matter in enhancing the perceived benefits of online health information sites? Application of the updated DeLone and McLean Information Systems Success Model. International Journal of Medical Informatics, 137-143. doi:https://doi.org/10.1016/j.ijmedinf.2020.104093

Solano, O., García, D., \& Bernal, J. (2014). Influence of the implementation of information systems on performance insmall and mid-sized enterprises: An empirical study in Colombia. Cuadernos de Administración. 30(52), 31-43.

Song, J., Migliaccio, G., Wang, G., \& Lu, H. (2017). Exploring the influence of system quality, information quality, and external service on BIM user satisfaction. Journal of Management in Engineering, 33(6).

Tamayo, M. (2007). El proceso de la investigación científica. Caracas. Venezuela: Editorial Limusa.

Tandon, U., Kiran, R., \& Sah, A. (2016). Analysing the complexities of website functionality, perceived ease of use and perceived usefulness on customer satisfaction of online shoppers in India. International Journal of Electronic Marketing and Retailing, 7(2), 115-140.

Utama, S., Purnomosidhi, B., \& Andayani, W. (2017). The Role of Perceived Usefulness on User Satisfaction of Regional Finance Information System. Jurnal Economia, 13(2), 141-154.

Wang, G., \& Song., J. (2017). The relation of perceived benefits and organizational supports to user satisfaction with building information model (BIM). Computers in Human Behavior, 68, 493500 .

$\mathrm{Xu}, \mathrm{F} ., \quad \& \mathrm{Du}, \mathrm{J}$. (2018). Factors influencing users' satisfaction and loyalty to digital libraries in Chinese universities. Computers in Human Behavior, 83, 64-72.

Ynzunza, C., \& Izar, J. (2011). Efecto de las estrategias competitivas y los recursos y capacidades orienta-dos al mercado sobre el crecimiento de las organizaciones. Contaduría y Administración, 58(1), 169-197. doi:10.1016/S0186-1042(13)71202-6

Zaied, A. (2012). An integrated success model for evaluating information system in public sectors. Journal of Emerging Trends in Computing and Information Sciences, 3(6), 814-825. 